

\section{EVALUATION OF AN ION \\ PROPULSION SYSTEM \\ FOR A SYNCHRONOUS \\ SPACECRAFT MISSION}

Kenneth I. Duck Robert O. Bartlett

Robert J. Sullivan

AUGUST 1967

GODDARD SPACE FLIGHT CENTER

Greenbelt, Maryland

Submitted to AIAA Electric Propulsion and Plasmadynamics Conference September 11-13, Colorado Springs, Colorado 
EVALUATION OF AN ION PROPULSION SYSTEM FOR A

SYNCHRONOUS SPACECRAFT MISSION

Kenneth I. Duck, Robert O. Bartlett, and Robert J. Sullivan NASA-Goddard Space Flight Center

\begin{abstract}
An ion propulsion system has been investigated to determine its applicability for a synchronous spacecraft mission. The major objectives of the study have been to (1) determine the optimum thruster locations on the spacecraft, (2) establish thruster operational modes for both station keeping (North-South and East-West) and precise attitude control, and (3) obtain a definition of propulsion system sizing with regards to power and weight.
\end{abstract}

These studies were performed using a six-degree-offreedom mathematical model in conjunction with a hybrid computer and a Goddard Space Flight Center (GSFC) developed energy management-power system simulator. The simulation included the effects of (1) triaxial earth, (2) sun and moon perturbations, (3) real thruster characteristics, (4) solar radiation pressure, (5) gravity gradient torque, and (6) thrust beam vectoring.

Results from the studies indicate that the optimum thruster combination is composed of four beam vectored modules, three of which are in continuous operation. In addition, an ion propulsion system was evaluated in detail for a large communications spacecraft with regards to propulsion system weight, peak power requirement, average power requirement, thrust level, moment arm, and attitude duty cycle. Also presented are problem areas encountered in designing an ion propulsion system for the particular spacecraft, namely, thrust beam impingement and obtaining a large enough moment arm to minimize the thrust level, attitude duty cycle, and, consequently, power requirement. The analysis presented allows one to perform a preliminary total system sizing of an ion propulsion system for a synchronous mission and presents some insight into the problems that may be encountered.

\section{NOMENCLATURE}

$\vec{a}_{e}-$ Earth gravitational attraction on vehicle

$\vec{a}_{\text {SMS }}-$ Total acceleration placed on vehicle by sun and moon

$\vec{a}_{\text {SME }}-$ Total acceleration placed on Earth by sun and moon

$\vec{a}_{E}-$ Thrust acceleration

g - Acceleration of gravity

$\overrightarrow{\mathrm{h}}$ - Vehicle angular momentum vector

I - Moment of inertia
J - Inertia dyadic

$I_{\text {sp }}$ - Specific impulse

$I_{T}-$ Total impulse

$\mathrm{k}_{\mathrm{T}}$ - Tankage structural factor

$\overrightarrow{\mathrm{M}}_{c}$ - Control torque

$M_{D}$ - General disturbance torque

$\overrightarrow{\mathrm{M}}_{\mathrm{GG}}$ - Gravity gradient torque

$\overrightarrow{\mathrm{M}}_{\mathrm{sp}}-$ Solar torque

$\overrightarrow{\ddot{r}}$ - Total vehicle translation acceleration

$t_{B}-$ Thrust time in soft limit cycle

$t_{c}$ - Coast time in soft limit cycle

T - Limit cycle period

$\Delta \mathrm{V}$ - Velocity increment

$W_{0}-$ Vehicle total weight

$w_{p}-$ Propellant weight

$W_{\text {power }}$ - Solar array and battery weight

$W_{p p}$ - Total propulsion and power system weight

$W_{p s}-$ Propulsion system weight

$\mathrm{W}_{\mathrm{T}}$ - Propellant tankage weight

$\theta_{\mathrm{DB}}-$ Vehicle attitude dead-zone angle

$\Delta \dot{\theta}$ - Change in vehicle angular rate

$\Delta \theta-$ Change in vehicle attitude angle

$\sigma$ - Attitude duty cycle

$\vec{\omega}-$ Total vehicle angular velocity vector.

\section{INTRODUCTION}

The maintenance of attitude control and station keeping of equatorial Earth synchronous space vehicles has been the subject of many papers during the last decade. The primary purpose of this paper, as has been the purpose of other papers, is to examine these functions using ion engines as the thrust actuators. In this paper, the 
thrust and power requirements have been studied in general for a spectrum of vehicle sizes (500-10,000 pounds) and in much detail for an advanced communications vehicle of the 1500 pound class. In the detailed studies such items as thruster operational modes, attitude control duty cycle, and problem areas were examined.

\section{SLMULATION MODEL}

The studies presented in this paper were generated using both analytical and simulation techniques. The simulation consisted of a six degree-of-freedom dynamical model programmed on a hybrid computer system which was interfaced with a GSFC developed electric propulsion energy management-power system simulator.

The dynamical model was composed of the translational equations based on Newton's second law coupled with the Eulerian equations describing the motion of a rigid body about its mass center. The simulation model included the effects due to (1) triaxial earth, (2) solar and lunar gravitational interaction, (3) solar radiation pressure, (4) gravity gradient torque, and (5) thrust beam vectoring. The general equations of motion can be written in vectorial form as

$$
\overrightarrow{\ddot{r}}=\vec{a}_{E}+\left(\vec{a}_{\text {SMS }}-\vec{a}_{\text {SME }}\right)+\vec{a}_{T}
$$

and

$$
\mathrm{J} \overrightarrow{\dot{\alpha}}+\overrightarrow{\mathrm{u}} \times \overrightarrow{\mathrm{h}}=\overrightarrow{\mathrm{M}}_{\mathrm{GG}}+\overrightarrow{\mathrm{M}}_{\mathrm{sp}}+\overrightarrow{\mathrm{M}}_{\mathrm{c}}
$$

The system used for determining the vehicle attitude was that of Euler angles. The rotational sequence and the gravity torque model used are those found in reference 1. The triaxial earth model used is the fourth order method presented in reference 2. The sun and moon gravitational interaction was treated as two forces resulting from bodies with spherical symmetry and their respective inertial locations were computed using Newcomb's Equations. (3)

The hybrid computer system consists of an analog computer coupled through appropriate interface to a digital computer to utilize their respective computational advantages in solving a problem. The system used for the studies presented here consists of an EAI $231 \mathrm{R}-\mathrm{V}$ general purpose analog computer, EAI DOS-350 digital operations system, and SDS-9300 general purpose digital computer all coupled by a specially designed interface. The interfacing between the analog and digital systems is achieved by an analog-to-digital converter with a twelve channel multiplexer, six digital-to-analog converters, sample interval timer, and an analog slave control. The interfacing between the digital computer and the digital operations system consists of sense lines, interrupts and twenty-four bit word digital-to-digital transfer equipment. Figure 1 presents a block diagram of the hybrid computation system.

The electric propulsion energy management-power system simulator was developed due to the need and desirability of having a simulation tool which would permit system studies of electric thrusters as space vehicle

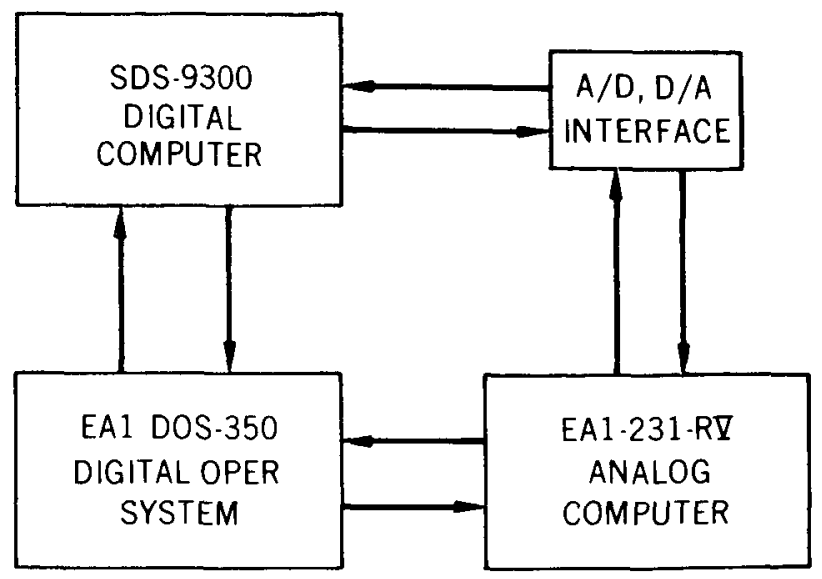

Figure 1-Hybrid Computer System

attitude control and station keeping actuators. This simulator makes it possible to "fly" a space mission in the laboratory. Parameters such as vehicle attitude, orbit, solar illumination, and environmental perturbations are programmed into the simulator and the thruster system under study, including its power source, then undergo the same demands as would be placed upon it in an actual mission. The major elements of the simulator are (1) power source, (2) energy storage unit and (3) programmable power load.

The power system simulated for this paper is of the solar electric type. The solar array output was assumed to be primarily a function of the angle made by the array and the incident sun light and the temperature of the array. This assumption is prefaced by establishing the design of the solar array, the solar radiation intensity, and the array's aging factor due to particle radiation damage. The solar array system was simulated using a programmable laboratory power supply which was programmed according to the angle of incidence and the array temperature generated by the computer dynamical simulation. The energy storage unit consists of flight quality Ni-Cd spacecraft batteries. The programmable load dissipates electrical energy from the source-storage system with respect to the thrust level and the vehicle dynamics as generated by the computer system. This simulator system is modular which allows the evaluation of other types of power systems with a minimum of set-up time. For example, a $\mathrm{Ag}-\mathrm{Cd}$ battery system could be used by simply exchanging the respective battery banks, or an RTG power source simulated by using the proper programming techniques. In addition, this modular approach allows the integration of flight hardware into the simulator system thus allowing an empirical system evaluation of electric thruster performance. Figure 2 presents a block diagram of the system.

The total simulation required coupling of the dynamical model with the computation and electric propulsion power system simulator. This was achieved by integrating the translational equations on the digital computer and the rotational equations on the analog computer. The attitude control actuation logic was generated by the digital operations systems. Figure 3 presents a block diagram of the total simulation. 


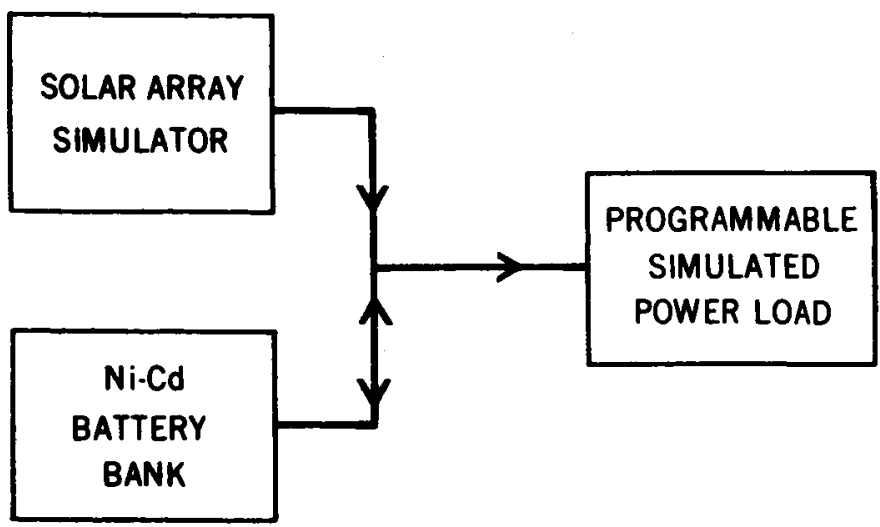

Figure 2-Electric Propulsion - Energy ManagementPower System Simulator

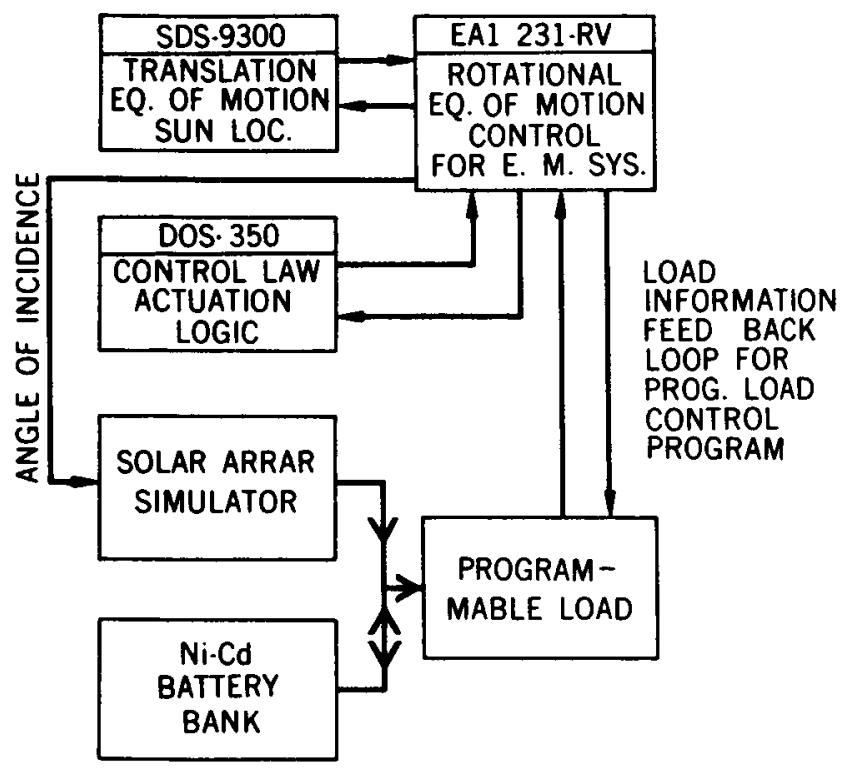

Figure 3-Total Simulation Block Diagram

\section{STATION KEEPING AND ATTITUDE CONTROL USING ION ENGINES}

\section{Station Keeping}

There are two primary sources of disturbances which cause a 24 hour synchronous space vehicle to drift from its desired station. These sources are (1) the nonspherical or triaxial structure of the Earth and (2) the interaction of the gravitational fields of the sun and moon. These disturbances have been examined in great detail $(2,4)$ and only a cursory discussion of their respective effects will be given here.

The triaxial Earth affects the motion of the vehicle both in and out of the orbit plane. The in-plane or EastWest (E-W) effect has the tendancy to make the vehicle drift or slide in longitude with respect to a station on the rotating Earth. The out of plane motion resulting from the triaxial earth is negligible.(2) The amount of E-W drift depends upon the longitude chosen for the operating station. There exist four quasi "stable" longitudes, i.e. positions where no additional energy is required for station keeping. These points lie on extensions of the minor and major axes of the equator. The points on the minor axis are the only true stable positions. A vehicle injected near these points if uncorrected tends to oscillate about the point with small damping and thus tends to stabilize on the point $(2,4)$. As for the points on the major axis, a disturbance can cause the vehicle to oscillate about the point through an amplitude of $\pm 90^{\circ}$ with a period of about 3 years. (2) It should also be noted that the maximum E-W perturbation occurs at longitudes located 45 degrees from the quasi "stable" longitudes.

The gravitational fields of the sun and moon affect the motion of an equatorial 24 hour synchronous spacecraft by causing a small oscillation of the radius vector and longitude and by inducing a small but continuously growing inclination of the vehicle orbit. The $\mathrm{E}-\mathrm{W}$ motion averages out to be nearly zero and is negligible compared to the E-W disturbance induced by the triaxial earth. The inclination induced however causes the vehicle to make a "figure eight" motion about the equator of the rotating earth. The maximum latitude excursion due to this effect is twice the value of the inclination. This inclination grows at a mean rate of approximately $0.9^{\circ}$ per year.

There are basically two methods of correcting the previously discussed station perturbations. They are: (1) let the vehicle drift off station and use impulsive maneuvering to re-position the vehicle; or (2) size the thrust level such that it is equal to the mean station perturbation and directed in the opposite sense. For ion engines the latter method is superior to the former since a smaller thrust level is required.

To counter balance the orbit perturbations resulting from the gravitational attraction of the sun and moon requires that the corrective thrust be directed normal to the orbit plane. It can be shown from the Gauss equations of planetary motion ( 6 ) that the most efficient method of $\mathrm{N}-\mathrm{S}$ station keeping is to supply an impulse at the line of nodes of the orbit. The efficiency of this maneuver decreases as the vehicle moves away from the nodes. It has been shown ( 5 ) that $\mathrm{N}-\mathrm{S}$ station keeping using ion engines is most efficiently performed in terms of total power and propulsion system weight by supplying a continuous thrust, the direction of which is reversed every half orbit. Such a system, as for the E-W case, imposes the requirement of a lower thrust level and consequently smaller system weight and peak power level.

Design curves have been generated from which one can size the thrust level, power requirement, and system hardware weight for ion engine station keeping systems operating in a continuous thrusting mode.

Figure 4 is a plot of the E-W station keeping yearly $\Delta V$ requirement as a function of geographic longitude. Figure 5 is a plot of $\mathrm{E}-\mathrm{W}$ station keeping thrust level as a function of spacecraft weight using a continuous thrusting mode. Figure 4 is to be used in conjunction with Figure 5 to determine the E-W station keeping thrust requirement. 


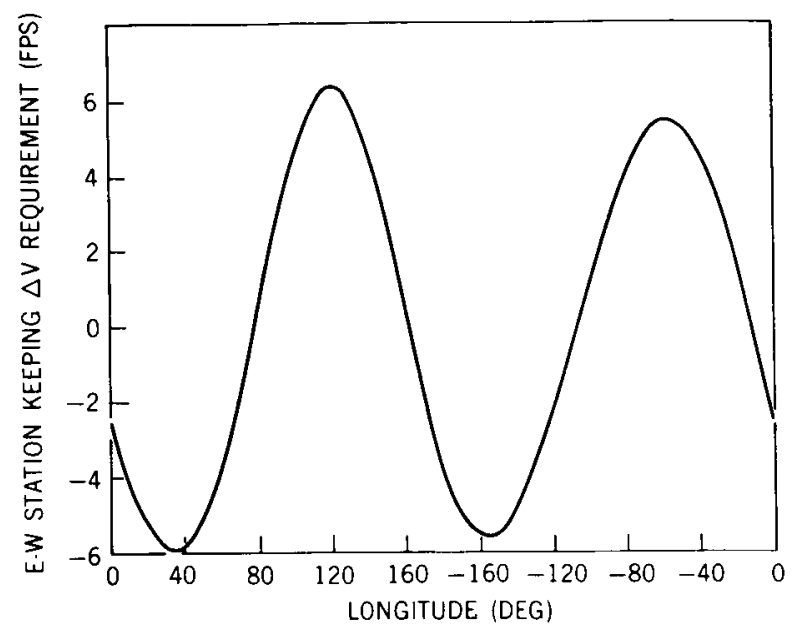

Figure 4-E-W Station Keeping Yearly $\Delta \mathrm{V}$ Requirement

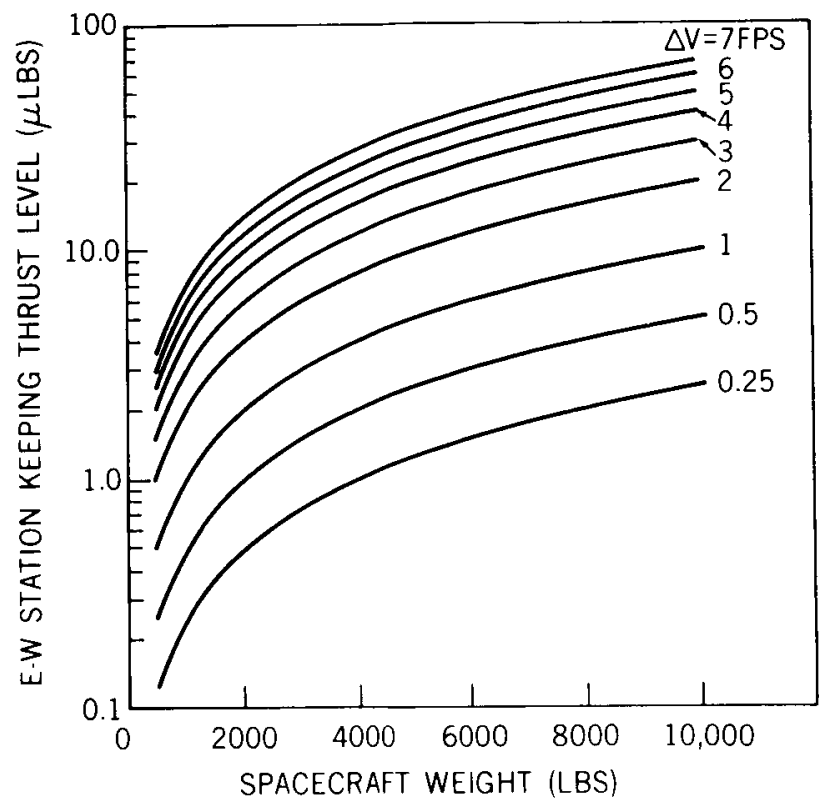

Figure 5-E-W Station Keeping Thrust Level Using A Continuous Thrusting Mode

Figure 6 is a plot of the N-S station keeping thrust level as a function of vehicle weight.

Figure 7 is a plot of the power required from the power generation source as a function of engine thrust level (7) the ion engines shown are the Cesium bombardment and the Cesium contact types.

Figure 8 is a plot of engine hardware weight per thruster excluding fuel and tankage for the previously mentioned thrusters as a function of thrust level. This figure is based upon the empirical formulae presented in reference. ${ }^{(8)}$ The power conditioning system is based on a system source power less than $2.5 \mathrm{KW}$.

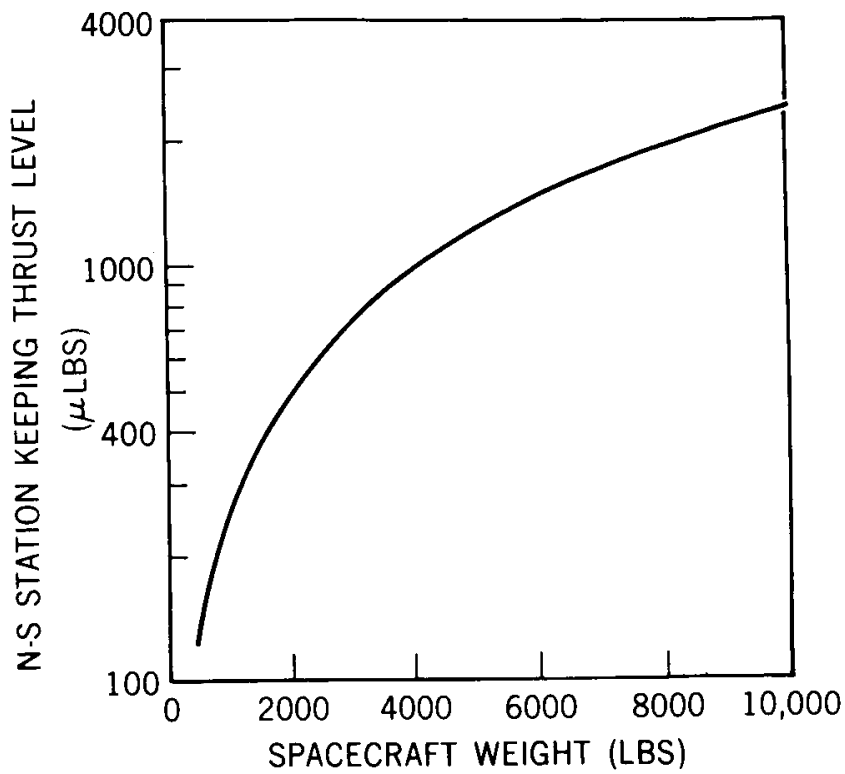

Figure 6-N-S Station Keeping Thrust Level Using A Continuous Thrusting Mode

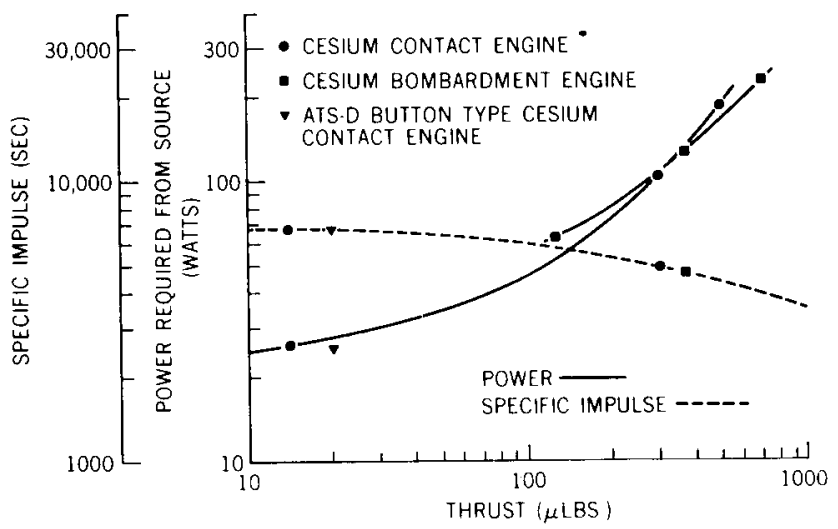

Figure 7-Power Required From Source and Specific Impulse as Functions of Thrust For Cesium Ion Thrusters.

If the total impulse or the velocity increment requirement for a particular mission is known, the propellant weight can be found from one of the two following equations.

$$
W_{p}=\frac{I_{T}}{I_{s p}}
$$

or

$$
W_{p}=W_{0}\left(1-e^{-\Delta V / g I s p}\right)
$$

The tankage weight is a function of the fuel weight and can be written as 


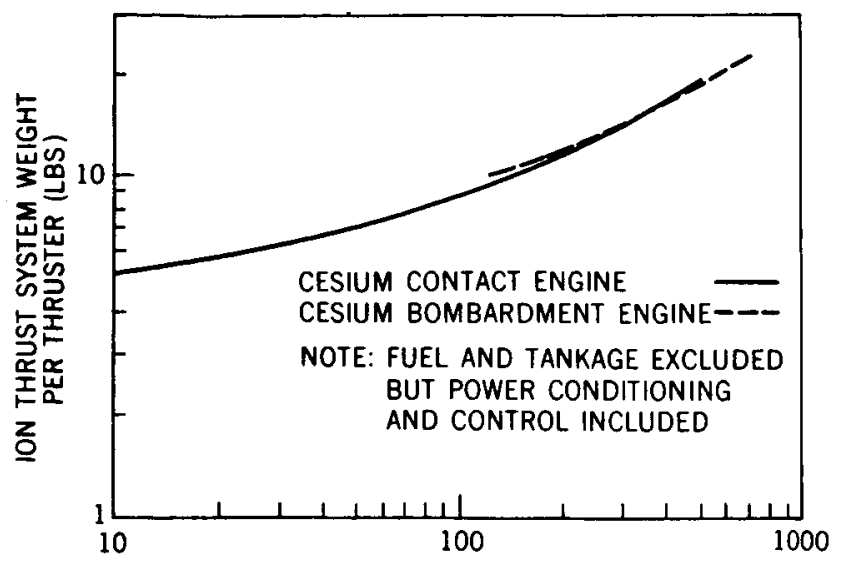

THRUST $(\mu$ LBS $)$

Figure 8-Ion Thrust System Weight Per Thruster For Cesium Ion Thrusters

$$
w_{T}=k_{T} w_{p}
$$

where .67 $\leq k_{T} \leq 1$ depending upon whether the fuel load is large or small respectively. (8)

Using the design curves presented in conjunction with the above equations, one can perform a quick assessment of station keeping thrust levels, power requirements, and propulsion system weight (excluding power source and energy storage devices) for a particular spacecraft.

\section{Attitude Control}

The attitude control system function is to hold a prescribed orientation of the vehicle within specified limits. This system must have the ability to control the vehicle attitude in the presence of such natural effects as solar radiation pressure, gravity gradient torque, magnetic fields, micrometeorite impact and such internal effects as thrust vector misalignment, moving parts and vehicle out-gassing.

To control the attitude of vehicle using reaction jets requires that thrust be directed in at least six different directions. Using ion engines, attitude control can be achieved using one of two methods: (1) having 2 equal but opposing jets for each vehicle rotation axis or (2) use of an electrostatic field to vector the thrust beam of the previously described station keeping thrusters. The six jet system offers the advantage of lower thrust level since the jets can be optimally located for attitude control (i.e., normal to the moment arm). However, a higher system weight could result since additional station keeping thrusters would be required. This means that a total of nine jets would be required to achieve both attitude control and station keeping compared to the beam vectored system where both functions can be achieved using four jets. Also the nine jet system requires a warm-up and shutdown period and the limit cycle period must be sized accordingly along with the inclusion of a complex lead-lag logic in the control system electronics. The thrust pulses for the beam vectored system are almost square waves with the system time constants in the microsecond range. Both types of systems will be sized and discussed in the following section for a particular spacecraft model.

The concept of beam vectoring in a soft limit cycle has been studied in some detail in the Appendix. The Appendix assumes that cross coupling can be ignored since an ion propulsion system can only control small rates in the limit cycle operation. Using the equations developed in the Appendix, the maximum rate in the limit cycle has been calculated for a range of dead zones between 0.1 and 0.5 degrees and for a range of disturbance accelerations between $10^{-11}$ and $10^{-5} \mathrm{rad} / \mathrm{sec}^{2}$. These studies indicate that the maximum rate that can be controlled in a soft limit cycle decreases as the beam vectoring or attitude duty cycle increases. Figure 9 is a plot of the maximum rate in the soft limit cycle as a function of maximum angular acceleration and attitude duty cycle for a dead zone angle of \pm 0.1 degree. Figure 9 indicates that the maximum rate controlled approaches zero very rapidly for duty cycles greater than 80 per cent. This same trend was borne out by similar studies for other dead zone sizes. The significance of this study is that in designing a system for attitude control using beam vectoring techniques on the station keeping engines, one should size the attitude duty cycle such that it will be equal to or less than 80 percent at the peak disturbance torque. It has been assumed for all cases that a coarse attitude control system is present on board the spacecraft to place the vehicle rotational dynamics in a range that can be controlled by the ion engine system.

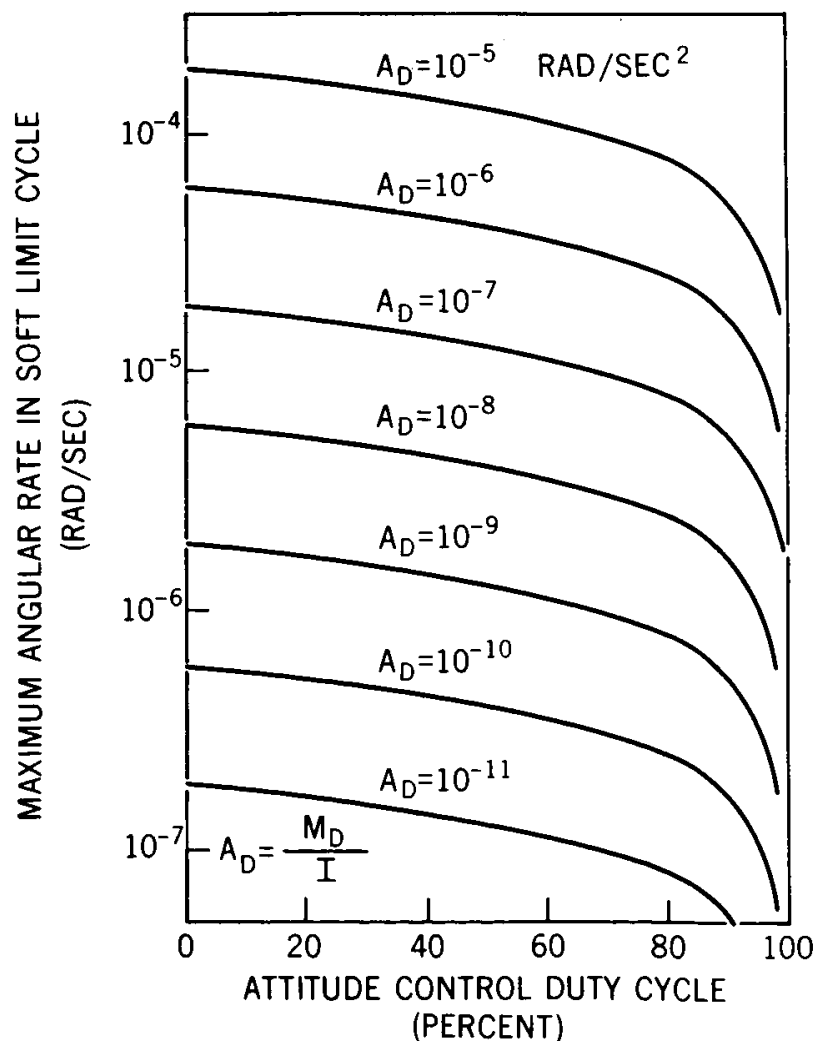

Figure 9-Maximum Soft Limit Cycle Rate as a Function of Disturbance Acceleration and Attitude DutyCycle 
station keeping jets and (2) use of the same jets for both attitude control and station keeping functions. It was assumed that all station keeping systems are continuous. thrust systems sized such that their thrust level is equal but opposite to the average perturbations acting on the vehicle orbit. Relative to the two basic concepts a total of six configurations were examined and will be subsequently discussed. It was assumed for all cases that the station keeping engines thrust through the vehicle C.M.

Configurations I and II both utilized separate attitude control and station keeping jets. These configurations differ only in the location of the attitude control jets, i.e. configuration I has the six attitude control and three station keeping jets attached to the aft module while configuration II has the attitude jets attached to the edge of the parabolic antenna to increase the moment arm. These configurations are shown in Figures 11 and 12 respectively. the attitude sensors. This module is attached to the antenna by a strut structure. The antenna itself is attached to the equipment module which faces away from the Earth. This module will be referred to as the aft module.

The electrical power source consists of solar cells attached to both sides of each of four paddles. These paddles are attached to the spacecraft by four reinforced struts such that the face of two paddles is parallel to the antenna face and the remaining two such that they are normal to the antenna face.

It should be noted that the antenna gain degrades quite significantly if precise attitude control and station keeping are not provided. It should also be noted that a coarse propulsion system is required for initial station acquisition, any subsequent slewing maneuvers to other ground stations, and any large orbit changes because the high power required by an ion thrust system to perform these maneuvers in a comparable period of time is prohibitive. Figure 10 is a conceptual drawing of the spacecraft.

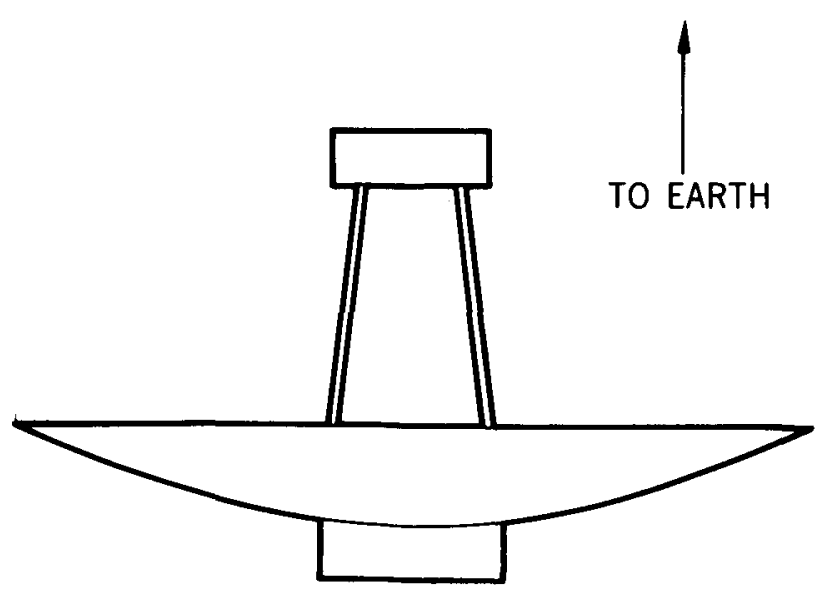

Figure 10-Advanced Communications Spacecraft

In order to size and design an ion propulsion system for precise attitude control and station keeping of the described spacecraft, two basic concepts have been examined. They are (1) use of separate attitude control and

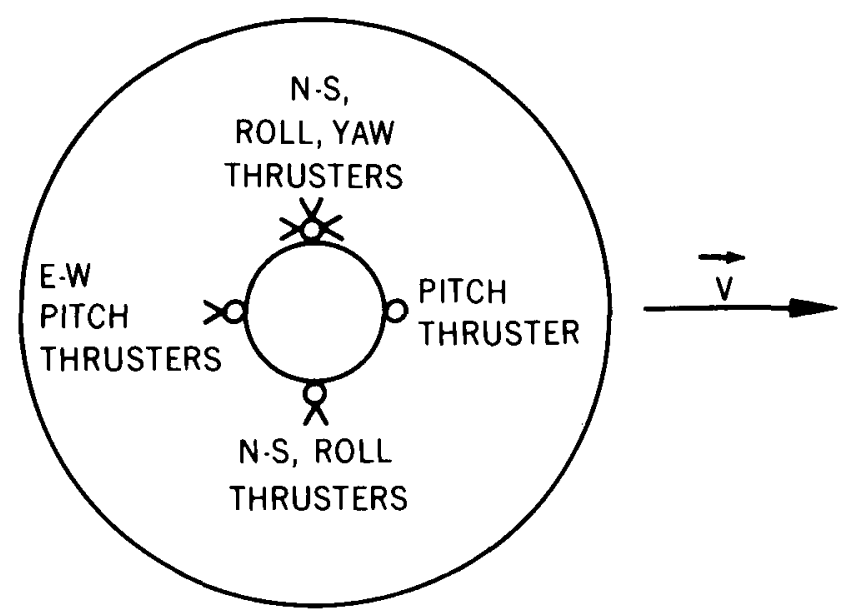

NOTE: LOCAL VERTICAL NORMAL TO PLANE OF FIGURE

Figure 11-Thruster Configuration I

YAW AND ROLL THRUSTERS

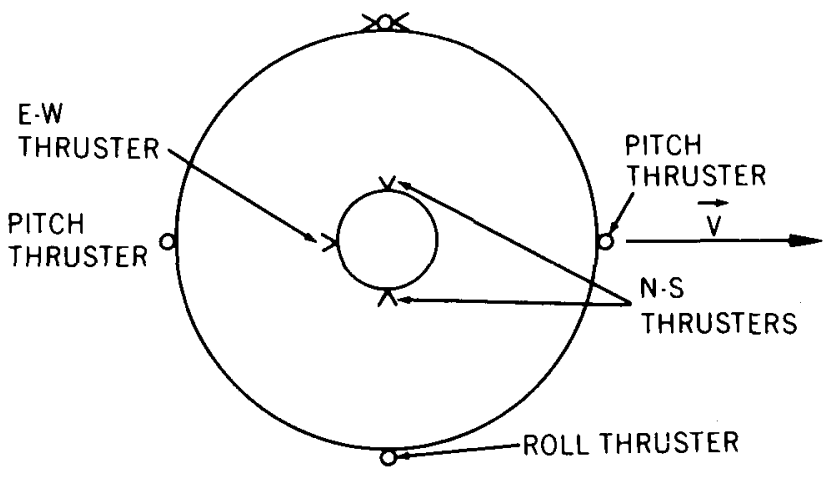

NOTE: LOCAL VERTICAL NORMAL TO PLANE OF FIGURE

Figure 12-Thruster Configuration II

Configurations III through VI utilize electrostatic vectoring of the station keeping engine thrust beams to generate an altitude torque. 
Configuration III consisted of two jets mounted such that one jet applied a thrust component in the orbit plane and the other out of the plane. This system was eliminated from further analysis for two reasons; (1) the maximum thrust level required for E-W station keeping for the 1500 pound spacecraft (10 micropounds) was found to produce inadequate control torque when beam vectored and (2) in order to accomplish $\mathrm{N}-\mathrm{S}$ station keeping using a continuous thrusting mode it is necessary to thrust in opposite directions over half of an orbit, a direct contradiction with regards to the E-W jet (note also that redundant sensors would also be required).

Configuration IV was similar to configuration III except that two jets were used, each on over half the orbit, for N-S station keeping. This configuration was also eliminated due to insufficient attitude torque when beam deflecting the E-W station keeping thruster.

Configurations V and VI both consisted of four beam vectored modules of which three are in a continuous thrusting mode. Two of the modules produce their undeflected thrust beams in the orbit plane and are so situated that the thrust level of one bias the other through the vehicle C.M. to obtain the small $\mathrm{E}-\mathrm{W}$ translational thrust and yet when beam vectored produce sufficient attitude torque about the pitch and yaw axes.

The other two modules are located such that their respective undeflected thrust beams pass through the vehicle C.M. and are each fired separately over a half orbit to provide $\mathrm{N}-\mathrm{S}$ station keeping and attitude control about the roll axis. Configuration $\mathrm{V}$ has the thruster modules attached to the aft module while configuration VI has them attached to the edge of the parabolic antenna. Configurations $\mathrm{V}$ and VI are presented by figures 13 and 14 respectively.

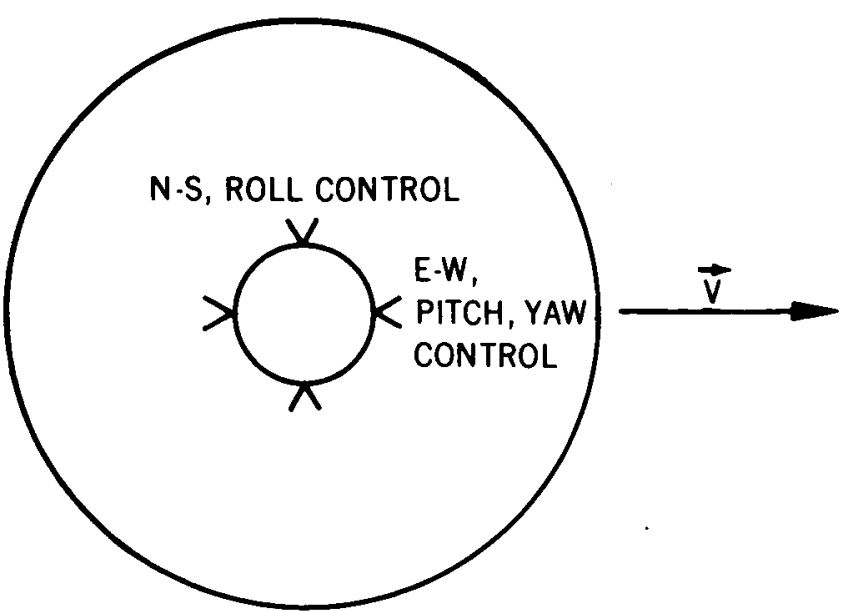

NOTE: LOCAL VERTICAL NORMAL TO PLANE OF FIGURE

Figure 13-Thruster Configuration V

Table I presents a synopsis of the configurations examined. The system weight and power data presented in Table $I$ are based on an attitude duty-cycle of 80 percent under maximum torque conditions. It should be noted that the duty-cycle of 80 percent applies to only the pitch and yaw axes for the beam vectored systems since the

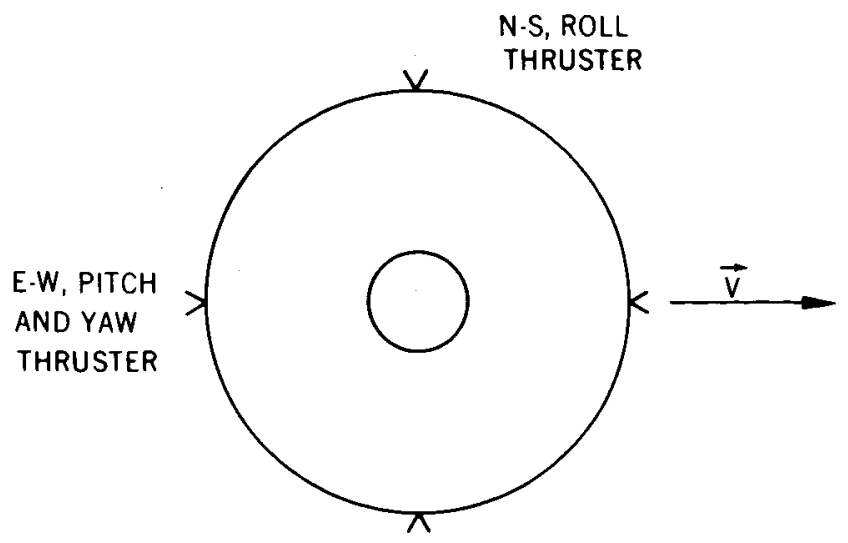

NOTE: LOCAL VERTICAL NORMAL TO PLANE OF FIGURE

Figure 14-Thruster Configuration VI

large N-S station keeping thrust level (i.e. large relative to the E-W requirement) imposes a duty-cycle about the roll axis. The primary contributor of attitude disturbances on such a spacecraft as is studied here is solar radiation pressure torque, the maximum value of which is estimated to be approximately 200 micropound-feet.

Table I indicates that configurations I and $\mathrm{V}$ have larger system weights and power requirements than $\amalg$ and VI respectively. Note also that a thrust beam impingement problem exists for configuration $\mathrm{V}$ when in a beam deflected state in the direction of the antenna. Assuming that the thrust beam makes a cone having a 15 degree half angle and can be vectored \pm 20 degrees, it was found for configuration $\mathrm{V}$ that the thruster modules must be located at least 4.31 feet from the dish. This means that the aft module would have to extend even further than the 4.31 feet to distribute the vehicle mass such that undeflected thrust vector would pass through the center of mass. This creates a problem of fitting state-of-the-art launch vehicle shrouds. As a result of the small moment arm, heavy propulsion system weight, and large power requirement, configuration I will be eliminated from further study and configuration $\mathrm{V}$ will be eliminated for the same reasons along with the thrust beam impingement problem.

Configurations II and VI have been studied and compared in detail. The results of this study will now be presented.

The propulsion system hardware weight, peak and average power requirement, and average attitude dutycycle have been determined as a function of the maximum attitude duty-cycle (i.e. the duty-cycle under maximum disturbance torque conditions). For all cases the maximum duty-cycle was assumed to be equal to or less than 80 percent since it has been established in a previous section of this paper that the maximum angular rate controlled by the system approaches zero very rapidly beyond 80 percent. The hardware weight includes the thruster assembly, fuel and tankage for two years and the necessary power conditioning and control equipment. 
$\sigma_{M}=80 \%$

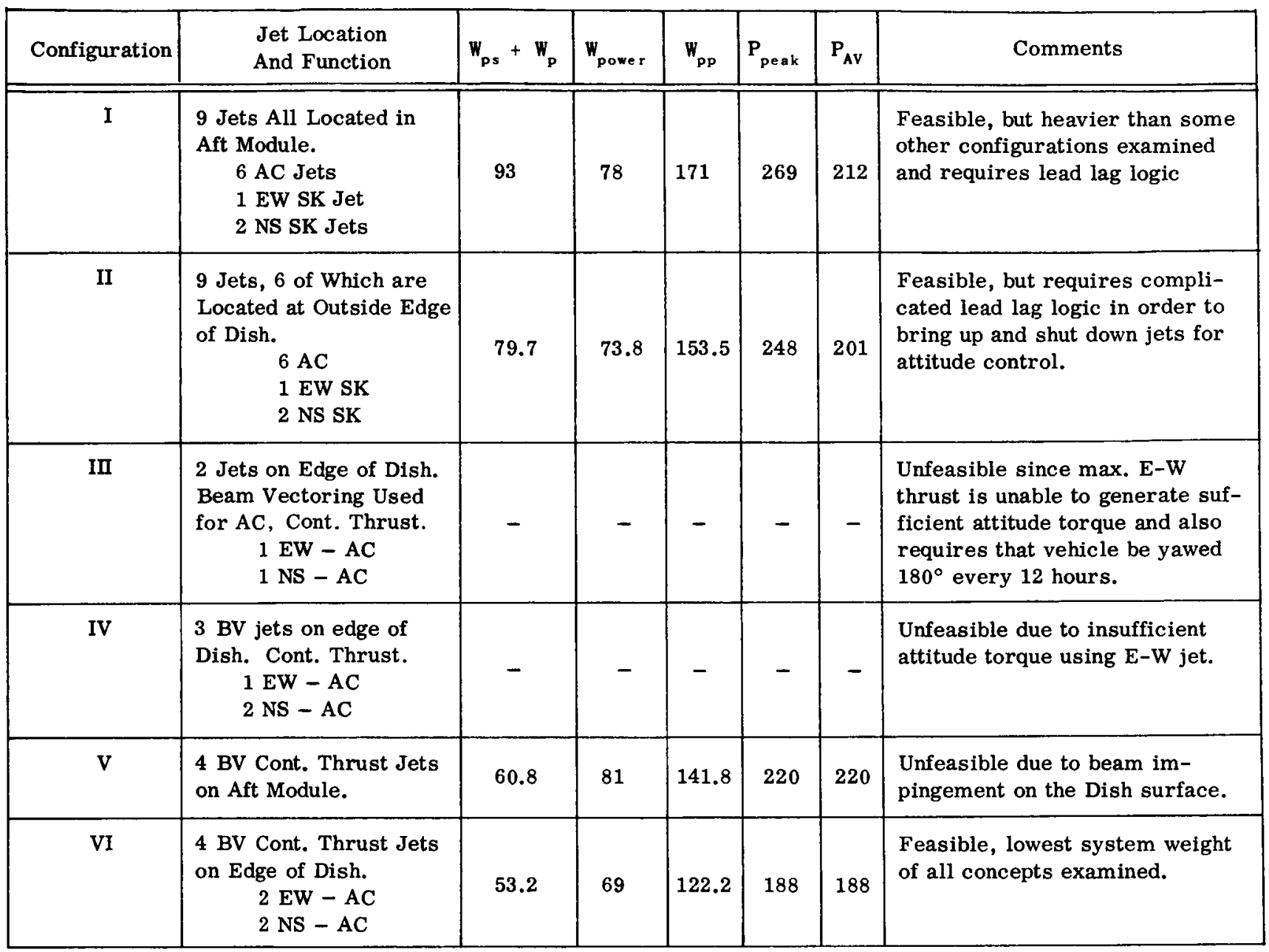

Figure 15 is a plot of propulsion system weight and average attitude duty-cycle as functions of the maximum duty-cycle. Figure 15 indicates that configuration VI is superior to configuration II in terms of hardware weight over the entire duty-cycle spectrum studied.

Since ion thruster systems consume more electrical energy than any other existing propulsion systems, it is necessary that the amount of power required (both peak and average) and the weight of any power producing equipment be determined. The power producing s,ist in assumed for this paper is a solar-electric syitem consisting of solar cells and nickel-cadamium batteries. The specific power assumed for the solar cell array is 4 watts per pound as an end of life performance level. The battery specific energy was assumed to be 10 watt-hours per pound.

Figure 16 presents both the peak and average power requirements for both configurations $\mathrm{II}$ and $\mathrm{VI}$ as a function of the maximum attitude duty cycle. Figure 16 indicates that the peak power requirement grows exponentially as the maximum duty cycle decreases for both systems. This results from the increased thrust level required to decrease the duty-cycle. The average power required by configuration II is less than that required by configuration VI for maximum duty-cycles below 62 percent.

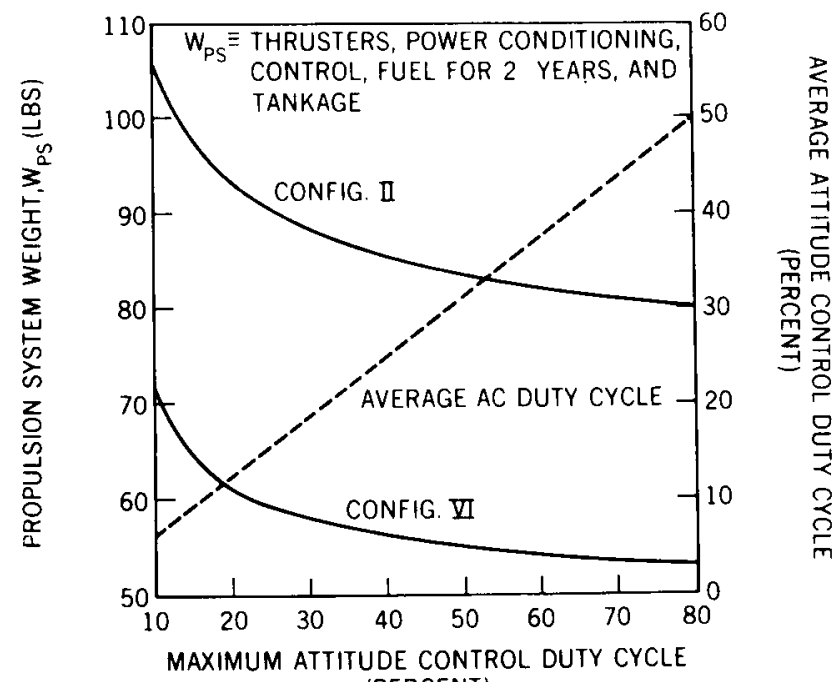

(PERCENT)

Figure 15-Propulsion System Weight And Average Attitude Control Duty-Cycle As Functions of Maximum Attitude Control Duty-Cycle 


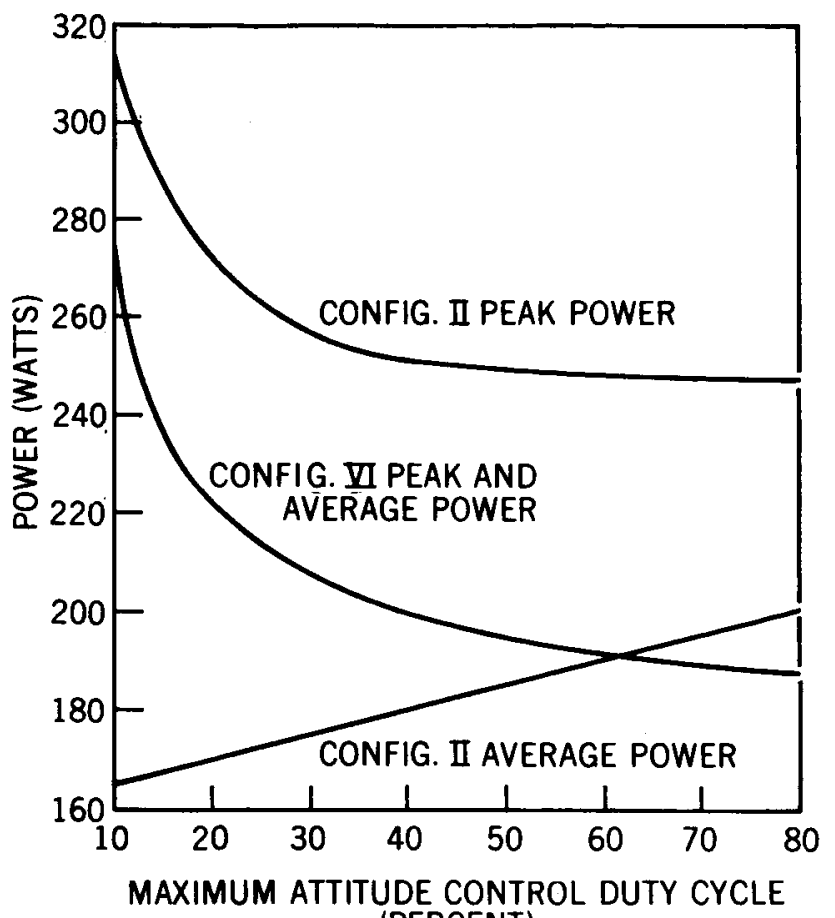

(PERCENT)

Figure 16-Peak And Average Power As A Function of Maximum Attitude Control Duty-Cycle (Percent)

The power system weight was determined for both configurations II and VI. The battery requirement was sized on the basis of the average power dissipated over the maximum eclipse period of 1.167 hours for an Earth synchronous equatorial spacecraft. The weight of the power generation equipment was added to the propulsion system weight to obtain a complete basis of comparison for both systems being analyzed. Figure 17 presents the combined power and propulsion system weights for both configurations II and VI as functions of the maximum attitude control duty-cycle. Figure 17 indicates that configuration VI is superior to configuration II for maximum duty-cycles greater than 11 percent. The reason for the cross over point is due to the fact that configuration VI with it continuous thrusting mode requires that more average power be applied to the system and thus has a much larger solar array-battery weight than configuration II.

Configuration VI has one advantage over configuration II that has not been previously mentioned. This advantage is the fact that no lead-lag logic is required by the control system for beam vectored engines since the thrust beam can be vectored instantaneously. The requirement for the lead-lag network stems from the fact that to keep the six engines used by configuration $\Pi$ for attitude control in a ready state would be wasteful in terms of the electrical energy dissipated and would also impose a penalty on the solar cell and battery requirements of the system. It should be noted that the feasibility of lead-lag compensation is questionable for the small attitude tolerance $\left( \pm 0.1^{\circ}\right)$ required by such a spacecraft as described here because a very rapid response is necessary to ensure successful operation.

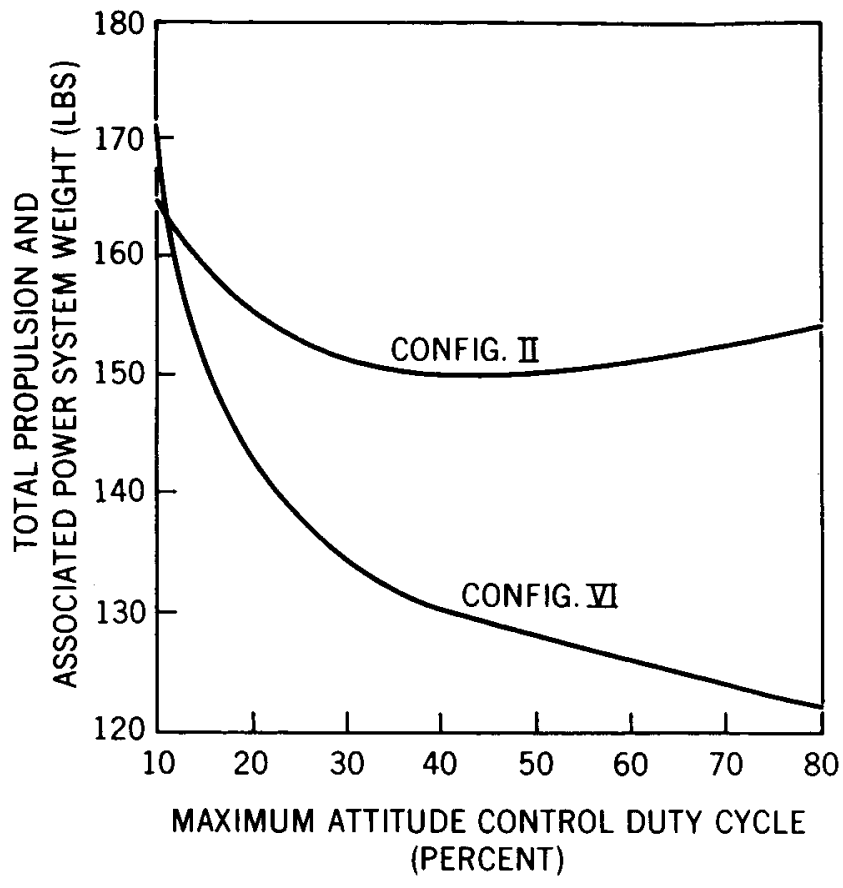

Figure 17-Total Propulsion and Associated Power System Weight as a Function of Maximum Attitude Control Duty-Cycle

In sizing a four module beam vectored ion thruster system there may exist several complications that require attention. Some of these problems are (1) thrust beam impingement on the vehicle structure, (2) beam deflection to generate an attitude torque about two axes, simultaneously, and (3) thruster module location such that the thrust level (and consequently power level and system weight) can be minimized and still generate an adequate control torque to offset the maximum expected attitude disturbance torque.

\section{CONCLUSIONS}

In conclusion it has been shown that an ion thrust system is applicable to Earth synchronous equatorial space vehicles requiring precise station keeping and attitude control. Performance curves were established from which a system definition in terms of weight and power can be determined for ion thrust systems. It was established that the attitude duty-cycle should be equal to or less than 80 percent under maximum disturbance torque conditions. Results from the specific system analysis indicated that a continuous thrust (beam vectoring attitude control) system was superior in terms of total system weight (i.e. thrust modules, power supply, energy storage, fuel, tankage, and power conditioning) to a system using separate attitude control and station keeping thrusters for maximum duty-cycles greater than 11 percent. Finally a group of critical problem areas were identified, some of which are complex lead-lag logic required for pulse mode systems, thrust beam impingement on the vehicle structure, generation of attitude torque about two axes simultaneously for the beam vectored system, and location of thrust modules such that the thrust level (and consequently, power requirement and weight) are minimized. 


\section{REFERENCES}

1. Barrett, C. C., "The Development of a Methematical Model and a Study of One Method of Orbit Adjust and Station Keeping Gravity-Oriented Satellites", NASA Technical Note D-3652.

2. Wagner, C. A., "The Drift of a 24-Hour Equatorial Satellite Due to an Earth Gravity Field Through 4th Order," NASA Technical Note D-2103.

3. Explanatory Supplement to the Astronomical Ephemeris and the American Ephemeris and Nautical Alamanac, Her Majesty's Stationary Office: London (1961).

4. Frick, R. H., and Gordon, T. B., "Perturbations of Synchronous Satellites," Rand Report R-399-NASA, The Rand Corp., Santa Monica, California (May, 1962).
5. Molitor, J. H., and Kaplan, M. H., "Optimization of Ion Engine Control Systems for Synchronous Satellites," Journal of Spacecraft and Rockets, Vol. 1 , No. 5 (Sept. - Oct., 1964).

6. Brouwer, D., and Clemence, G. M., Methods of Celestial Mechanics, Academic Press: New York and London (1961).

7. Mickelsen, W. R., "Advanced Concepts in Electric Propulsion," AIAA Paper No. 67-426 (July, 1967).

8. Shukay, T. R., "Power and Weight Requirements for Ion Engine Systems Applied to Specific Satellite Control Missions," AIAA Paper No. 66-498 (June, 1966).

\section{APPENDIX \\ Characteristics of a Soft Attitude Control Limit Cycle Using Beam Vectored Ion Engines}

The purpose of the derivation given below is to (1) determine the thrusting duty-cycle in the soft limit cycle operation as a function of the control and disturbance torques and (2) determine the dead zone size as a function of the maximum angular rate in the cycle, duty cycle, and disturbance acceleration. The derivation is applicable only for beam vectored ion engines since all thrust pulses are assumed to be perfect square waves. Also cross coupling is neglected based on the assumptions of small angular rates and small dead zone angles. plot.

Figure A-1 denotes a soft limit cycle phase plane

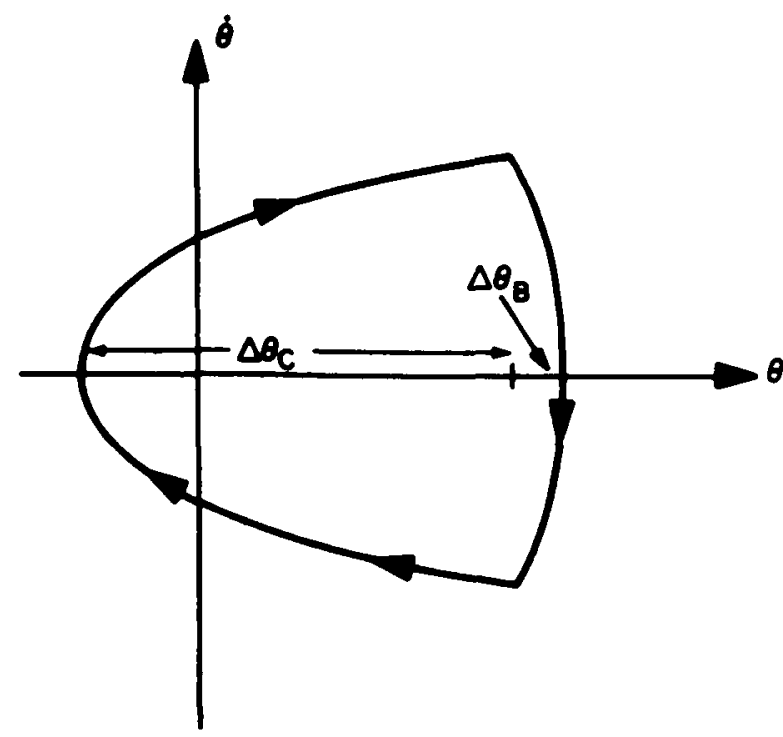

Figure A-1
During coast

$$
\left|\Delta \dot{\theta}_{c}\right|=\frac{M_{D}}{I} t_{c}
$$

and during beam vectoring

$$
\left|\Delta \dot{\theta}_{B}\right|=\frac{\left(M_{c}-M_{D}\right)}{I} t_{B}
$$

Note that for a steady state limit cycle operation

$$
\left|\Delta \dot{\theta}_{c}\right|=\left|\Delta \dot{\theta}_{B}\right|
$$

Substituting (A-1) and (A-2) into (A-3) yields

$$
\frac{t_{B}}{t_{c}}=\frac{M_{D}}{M_{C}-M_{D}}
$$

Assuming $M_{D}$ to be constant over a complete cycle

$$
\mathbf{T}=\mathbf{t}_{\mathrm{c}}+\mathbf{t}_{\mathrm{B}}
$$

Substituting (A-4) into (A-5) then

$$
T=\left[1+\frac{M_{D}}{\left(M_{C}-M_{D}\right)}\right] t_{c}
$$

Define the duty-cycle as

$$
\sigma=\frac{\mathbf{t}_{\mathbf{B}}}{\mathbf{T}}
$$


Substituting (A-4) and (A-6) into (A-7) and reducing yields

$$
\sigma=\frac{\mathbf{M}_{\mathbf{p}}}{\mathbf{M}_{\mathrm{c}}}
$$

Equation (A-8) then fulfills one of the objectives of this appendix as Eq. (A-8) defines the attitude duty cycle as a function of the disturbance and control torques.

Let

$$
A=\frac{\left(M_{c}-M_{D}\right)}{I}
$$

and

$$
A_{D}=\frac{M_{D}}{I}
$$

then during burn

$$
\begin{gathered}
\Delta \dot{\theta}_{B}=\mathbf{A} \mathbf{t}_{B} \\
\Delta \theta_{B}=\dot{\theta}_{O B} t_{B}+1 / 2 A t_{B}^{2}
\end{gathered}
$$

Eliminating time from $(A-11)$ and $(A-12)$

$$
\Delta \theta_{\mathrm{B}}=\frac{\dot{\theta}_{\mathrm{OB}} \Delta \dot{\theta}_{\mathrm{B}}}{\mathrm{A}}+1 / 2 \frac{\Delta \dot{\theta}^{2} \mathrm{~B}}{\mathrm{~A}}
$$

likewise during coast

$$
\Delta \theta_{c}=\frac{\dot{\theta}_{\alpha c} \Delta \dot{\theta}_{c}}{A_{D}}+1 / 2 \frac{\Delta \dot{\theta}_{c}^{2}}{A_{D}}
$$

Note from Fig. $(A-1)$ that

$$
\Delta \dot{\theta}_{\mathbf{C}}=-\Delta \dot{\theta}_{\mathbf{B}}
$$

and

$$
\dot{\theta}_{\text {oC }}=-\dot{\theta}_{\text {OB }}
$$

Define the deadzone angle, $\theta_{D B}$ as

$$
\theta_{\mathrm{DB}}=\left(\Delta \theta_{C}\right)_{\text {max }}+\left(\Delta \theta_{B}\right)_{\text {max }}
$$

Substituting (A-15), (A-16), into (A-14) and substituting the resulting equation along with (A-13) into $(A-17)$ and simplifying

$$
\theta_{\mathrm{DB}}=\dot{\theta}_{\mathrm{OB}} \Delta \dot{\theta}_{\mathrm{B}}\left(\frac{\mathrm{A}+\mathrm{A}_{\mathrm{D}}}{\mathrm{A}_{\mathrm{D}} \mathrm{A}}\right)+1 / 2 \Delta \dot{\theta}_{\mathrm{B}}^{2}\left(\frac{\mathrm{A}+\mathrm{A}_{\mathrm{D}}}{\mathrm{A}_{\mathrm{D}} \mathrm{A}}\right)
$$

Let

$$
\delta=\left(\frac{\mathbf{A}+\mathbf{A}_{\mathbf{D}}}{\mathbf{A}_{\mathbf{D}} \mathbf{A}}\right)
$$

Substituting (A-19) into (A-18) and rearranging

$$
0=\delta \Delta \dot{\theta}_{\mathrm{B}}^{2}+2 \delta \dot{\theta}_{\mathrm{OB}} \Delta \dot{\theta}_{\mathrm{B}}+2 \theta_{\mathrm{DB}}
$$

It is seen that equation $(\mathrm{A}-20)$ is a quadratic equation in terms of $\Delta \dot{\theta}_{B}$. To have any physical significance the discriminant of Eq. $(A-20)$ must be $\geq 0$. Then

$$
4 \delta^{2} \dot{\theta}_{\mathrm{OB}}^{2}-8 \delta \theta_{\mathrm{DB}} \geq 0
$$

Rewriting

$$
\begin{aligned}
\delta \dot{\theta}_{\mathrm{OB}}^{2} & \geq 2 \theta_{\mathrm{DB}} \\
\theta_{\mathrm{DB}} & \leq(\delta / 2) \dot{\theta}_{\mathrm{OB}}^{2}
\end{aligned}
$$

Substituting (A-8), (A-9), and (A-10) into (A-19) and rearranging

$$
\delta=\frac{I}{M_{D}} \frac{1}{(1-\sigma)}
$$

Substituting $(\mathrm{A}-23)$ into $(\mathrm{A}-22)$ yields

$$
\theta_{\mathrm{DB}} \leq \frac{\dot{\theta}_{\mathrm{OB}}^{2}}{2} \frac{\mathrm{I}}{\mathrm{M}_{\mathrm{D}}}\left[\frac{1}{(1-\sigma)}\right]
$$

It is now desirable to examine the validity of Eq. (A-24). Substitute $(A-8)$ into $(A-24)$ and solving for $\dot{\theta}_{0 B}^{2}$ yields

$$
\dot{\theta}_{\mathrm{OB}}^{2} \geq 2 \theta_{\mathrm{DB}} \frac{\left[\mathrm{M}_{\mathrm{D}} \mathrm{M}_{\mathrm{C}}-\mathrm{M}_{\mathrm{D}}^{2}\right]}{\mathrm{M}_{\mathrm{C}} \mathrm{I}}
$$

To have physical meaning then it is seen in Eq. (A-25) that

$$
\frac{M_{D} M_{c}-M_{D}^{2}}{M_{c} I} \geq 0
$$

thus

$$
M_{C} \geq M_{D}
$$

In examining Eq. (A-27) it is seen that if $\mathrm{M}_{c}=\mathrm{M}_{\mathrm{D}}$ control is not maintained since the vehicle does not move away from its initial position and would require that the attitude control torque source be on with a 100 percent duty-cycle. Therefore the necessary and sufficient condition to maintain control is

$$
M_{D}<M_{C}
$$

Thus the second objective of this appendix has been achieved in equation (A-24). 